\title{
Repetitive low intensity magnetic field stimulation in a neuronal cell line: A metabolomics study
}

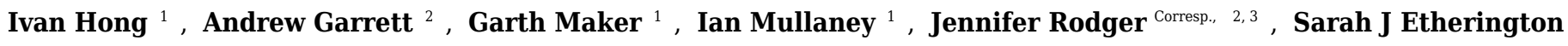 \\ ${ }^{1}$ School of Veterinary and Life Sciences, Murdoch University, Murdoch, WA, Australia \\ 2 School of Biological Sciences, Experimental and Regenerative Neuroscience, The University of Western Australia, Crawley, WA, Australia \\ 3 Brain Plasticity laboratory, Perron Institute for Neurological and Translational Science, Perth, WA, Australia \\ Corresponding Author: Jennifer Rodger \\ Email address: jennifer.rodger@uwa.edu.au
}

Low intensity repetitive magnetic stimulation of neural tissue modulates neuronal excitability and has promising therapeutic potential in the treatment of neurological disorders. However, the underpinning cellular and biochemical mechanisms remain poorly understood. This study investigates the behavioural effects of low intensity repetitive magnetic stimulation (LI-rMS) at a cellular and biochemical level. We delivered LI-rMS (10 $\mathrm{mT}$ ) at $1 \mathrm{~Hz}$ and $10 \mathrm{~Hz}$ to B50 rat neuroblastoma cells in vitro for 10 minutes and measured levels of selected metabolites immediately after stimulation. LI-rMS at both frequencies depleted selected tricarboxylic acid (TCA) cycle metabolites without affecting the main energy supplies. Furthermore, LI-rMS effects were frequency-specific with $1 \mathrm{~Hz}$ stimulation having stronger effects than $10 \mathrm{~Hz}$. The observed depletion of metabolites suggested that higher spontaneous activity may have led to an increase in GABA release. Although the absence of organised neural circuits and other cellular contributors (e.g. excitatory neurons and glia) in the B50 cell line limits the degree to which our results can be extrapolated to the human brain, the changes we describe provide novel insights into how LI-rMS modulates neural tissue. 
1 Repetitive Low intensity magnetic field stimulation in a neuronal cell line: a 2 metabolomics study

3

4 Ivan Hong 1,2, Andrew Garrett ${ }^{2}$, Garth Maker ${ }^{1}$, Ian Mullaney ${ }^{1}$, Jennifer Rodger 2,3, Sarah

5 J. Etherington ${ }^{1}$

6

7 1. School of Veterinary and Life Sciences, Murdoch University, Murdoch WA.

8 2. School of Biological Sciences, Experimental and Regenerative Neuroscience, University of 9 Western Australia, Crawley WA.

3. Perron Institute for Neurological and Translational Science, Nedlands, WA

Corresponding author:

Dr Jennifer Rodger

Experimental and Regenerative Neuroscience,

17 School of Animal Biology, University of Western Australia.

1835 Stirling Highway

19 Crawley 6009

20 Australia

21 Email: Jennifer.rodger@uwa.edu.au

22 Tel: $(+61) 864882245$

23 


\section{Abstract}

26 Low intensity repetitive magnetic stimulation of neural tissue modulates neuronal excitability and

27 has promising therapeutic potential in the treatment of neurological disorders. However, the

28 underpinning cellular and biochemical mechanisms remain poorly understood. This study

29 investigates the behavioural effects of low intensity repetitive magnetic stimulation (LI-rMS) at a

30 cellular and biochemical level. We delivered LI-rMS $(10 \mathrm{mT})$ at $1 \mathrm{~Hz}$ and $10 \mathrm{~Hz}$ to $\mathrm{B} 50$ rat

31 neuroblastoma cells in vitro for 10 minutes and measured levels of selected metabolites

32 immediately after stimulation. LI-rMS at both frequencies depleted selected tricarboxylic acid

33 (TCA) cycle metabolites without affecting the main energy supplies. Furthermore, LI-rMS effects

34 were frequency-specific with $1 \mathrm{~Hz}$ stimulation having stronger effects than $10 \mathrm{~Hz}$. The observed

35 depletion of metabolites suggested that higher spontaneous activity may have led to an increase in

36 GABA release. Although the absence of organised neural circuits and other cellular contributors

37 (e.g. excitatory neurons and glia) in the B50 cell line limits the degree to which our results can be

38 extrapolated to the human brain, the changes we describe provide novel insights into how LI-rMS

39 modulates neural tissue. 
43

4

45

\section{Introduction}

Faraday's discovery that a changing magnetic field induces a current in a conductor has contributed to many applications, such as the electromagnetic stimulation of body tissues and organs [Barker et al., 1985]. Specifically, electromagnetic stimulation of brain tissue has significant experimental and therapeutic potential because the induction of electric currents within neurons can modulate neuronal excitability, allowing non-invasive investigation and manipulation of brain circuit function and connectivity. Although clinical applications of magnetic fields most commonly involve high intensity fields that trigger action potentials and activate neural circuits [MüllerDahlhaus and Vlachos, 2013], therapeutic effects are also observed during low intensity magnetic stimulation [Di Lazzaro et al., 2013; Rohan et al., 2014]. For example in humans, low intensity repetitive transcranial magnetic stimulation (LI-rTMS; pulse amplitude $<100 \mathrm{mT}$ ) is beneficial in treating depression [Martiny et al., 2010] and pain [Shupak et al., 2004] and influences memory [Navarro et al., 2016].

It is apparent, however, that cellular mechanisms underpinning behavioural effects of LI-rTMS remain poorly characterised. Many studies in non-neuronal tissues and cells have focussed on the potentially negative effects of low intensity electromagnetic fields in the context of safety concerns surrounding the use of equipment emitting extremely low-frequency magnetic fields (ELF-MF; e.g. [ICNIRP, 1998]). However minimal investigation into low intensity stimulation in neuronal systems has been performed, despite demonstrated evidence of modulation of brain excitability in humans [Capone et al., 2009] and in animal models [Yang et al., 2015; Balassa et al., 2013]. It has been established that the intensity of the induced electric fields in LI-rTMS is not sufficient to depolarise neurons to action potential firing threshold [Davey and Riehl, 2006; Rudiak and Marg, 
1994]. In contrast, in vitro experiments have consistently shown that low intensity repetitive magnetic stimulation (LI-rMS- no cranium) modulates intracellular calcium levels in nonneuronal [Aldinucci et al., 2000; Walleczek and Budinger, 1992; Zhang et al., 2010] and neuronal cells [Grehl et al., 2015].

We recently demonstrated that LI-rMS of dissociated cortical neurons rapidly increases levels of intracellular calcium (within 10 minutes of the onset of stimulation), with higher levels of intracellular calcium detected following $10 \mathrm{~Hz}$ compared to $1 \mathrm{~Hz}$ stimulation [Grehl et al., 2015]. Such modulation of intracellular calcium alters NMDA receptor function [Manikonda et al., 2007] and provides a potential trigger for a wide range of changes in neuronal biochemistry which may underpin the LI-rTMS effects observed clinically [Martiny et al., 2010; Shupak et al., 2004]. Further, there is also evidence that low intensity magnetic fields alter levels of biochemicals that function in neuronal processes, for example, low intensity magnetic fields modulate the level of the primary metabolite of serotonin, 5-HIAA, in rat brain in a dose (time)-dependent manner [Shahbazi-Gahrouei et al., 2016]. In light of these findings, further investigation of biochemical and metabolic changes induced by LI-rMS in neuronal cells is warranted.

We hypothesize that changes in biochemical pathways due to LI-rTMS, will modify levels of a range of small molecule metabolites, including amino acids, carbohydrates and organic acids, which can be profiled using metabolomic techniques. Metabolomic analysis that profiles as many metabolites as possible in a single analysis is known as non-targeted screening. We performed such screening of a neuronal cell line immediately following 10 minutes of LI-rMS at $1 \mathrm{~Hz}$ or 10 
$88 \mathrm{~Hz}$ in vitro. We describe changes in the levels of 12 metabolites, 3 of which changed in a 89 frequency-dependent manner.

90

\section{METHODS}

92 Cell culture

93 Rat neuroblastoma cells from the B50 cell line were seeded directly onto 6-well plates and grown

94 for 24 hours in media containing DMEM with 5\% (v/v) heat-inactivated foetal calf serum, $2 \mathrm{mM}$ L-glutamine, $100 \mathrm{U} / \mathrm{ml}$ streptomycin and $100 \mathrm{U} / \mathrm{ml}$ penicillin. Cells were grown at $37^{\circ} \mathrm{C}$ within a $\mathrm{CO}_{2}$ incubator $\left(5 \% \mathrm{CO}_{2}+95 \%\right.$ air $)$. Cells from each 6 -well plate were later pooled during extraction to make one replicate. Each stimulus condition or control had 6 replicates in total.

8

\section{LI-rMS stimulation}

We used LI-rMS parameters that have previously been shown to increase intracellular calcium in primary cultured neurons in vitro [Grehl et al., 2015]. Stimulation was delivered to cells in the incubator using custom built round coils (34 mm diameter, $17.1 \mathrm{~mm}$ height, $0.812 \mathrm{~mm}$ thickness, 138 turns). In order to deliver reproducible stimulation to each well, coils were designed to fit within a single well of a 6-well plate so that a plate containing cells could be placed on top of a plate containing 5 coils, resulting in reliable and reproducible placement at a distance of $2.8 \mathrm{~mm}$ from the base of each well (Figure. 1A,B). As the stimulator could only accommodate 5 coils, only 5 wells were stimulated on each plate. The coils were driven by a $12 \mathrm{~V}$ magnetic pulse generator under control of a programmable micro-controller card (CardLogix, USA), which delivered monophasic pulses (rise time of $0.725 \mathrm{~ms}$ ). To approximate the stimulation dose in the 6-well plate, we measured the magnitude of the magnetic field in the $\mathrm{x}$ and $\mathrm{y}$ axes with a Hall Effect probe (Honeywell SS94A2D, USA). Magnetic field measurements were made at a distance of $0.5 \mathrm{~cm}$ 
112 from the base of the coil surface. Magnetic field strengths were converted to $\mathrm{dB} / \mathrm{dT}$, as a measure

113 of the change in magnetic field over time of each delivered pulse. A map of $\mathrm{dB} / \mathrm{dT}$ across the 6-

114 well plate was generated with Matlab 2013a (Figure 1C). Peak magnetic field and dB/dT inside

115 each well ranged between $\sim 2.2$ to $\sim 2.4 \mathrm{mT}$ and $\sim 3$ to $\sim 3.2 \mathrm{~T} / \mathrm{s}$ respectively. Coil temperature did

116 not rise above $37^{\circ} \mathrm{C}$, ruling out confounding effects of temperature change. Vibration of the coil

117 was assumed to be within vibration amplitude of background (bench surface), as shown previously

118 in Grehl et al.[2015]. For the experiments, 6-well plates were assigned to one of 3 conditions:

119 control (pulse generator switched off, no LI-rMS) $(n=6), 1 \mathrm{~Hz}$ (continuous stimulation for 10

120 minutes at $1 \mathrm{~Hz})(\mathrm{n}=4)$ and $10 \mathrm{~Hz}$ (continuous stimulation for 10 minutes at $10 \mathrm{~Hz})(\mathrm{n}=6)$.

121 Stimulation and sham (control) stimulations were performed at $37^{\circ} \mathrm{C}$ within the $\mathrm{CO}_{2}$ incubator.

122

\section{Metabolite extraction and derivatisation}

124 Cells were immediately quenched and washed twice post-stimulation with $2 \mathrm{ml}$ of ice-cold 125 phosphate-buffered saline. Cells were then scraped, aggregated and frozen in liquid nitrogen 126 before being freeze-dried. $500 \mu \mathrm{l}$ of extraction solution consisting of $2.6 \mu \mathrm{g} / \mathrm{ml}$ of ${ }^{13} \mathrm{C}_{6}$-sorbitol, as

127 an internal standard, was dissolved in methanol and added to the dried cells. Cells were lysed with

128 a Precellys 24 tissue lyser (Bertin Technologies, France) at 6500rpm for 40 seconds. The supernatant was evaporated before adding $500 \mu 1$ of water and freeze-drying again. Samples were 130 derivatised according to the protocol described in Abbiset al.[2012]. 20 $\mu 1$ of methoxyamine $\mathrm{HCl}$ solution $\left(20 \mathrm{mg} / \mathrm{ml}\right.$ in pyridine) was added to each extract and agitated at $1200 \mathrm{rpm}$ and $30^{\circ} \mathrm{C}$ for

13290 minutes. $40 \mu \mathrm{l}$ of $\mathrm{N}$-methyl-N-(trimethylsilyl) trifluoroacetamide (MSTFA) was subsequently 133 added to each extract and agitated at $300 \mathrm{rpm}$ and $37^{\circ} \mathrm{C}$ for 30 minutes. The derivatised samples 134 were transferred to $\mathrm{GC}$ vials and $5 \mu \mathrm{l}$ of alkanes $\left(\mathrm{C}_{10}, \mathrm{C}_{12}, \mathrm{C}_{15}, \mathrm{C}_{19}, \mathrm{C}_{22}, \mathrm{C}_{28} ; 0.156 \mathrm{mg} \cdot \mathrm{mL}^{-1} ; \mathrm{C}_{32}\right.$, 
$\left.135 \mathrm{C}_{36} ; 0.313 \mathrm{mg} / \mathrm{ml}\right)$ in hexane were added to each sample for calculation of a Kovat's retention

136 index, which aids comparison of data between samples.

137

138 Sample analysis

139 An Agilent 6890 series gas chromatograph with an Agilent 7863 autosampler coupled to an 140 Agilent 5973N single quadrupole gas chromatography-mass spectrometer(GC-MS) (Agilent 141 Technologies, Australia, Mulgrave, Australia) was used along with a Varian Factor Four-fused 142 silica capillary column VF-5ms $(30 \mathrm{mx} 0.25 \mathrm{~mm} \times 0.25 \mu \mathrm{m}+10 \mathrm{~m}$ EZ-Guard). The analytical 143 method was also as described in Abbisset al.[2012].1 $\mu 1$ of the sample was injected splitless into 144 the inlet that was set to $230^{\circ} \mathrm{C}$. The oventemperature was initially set to $70^{\circ} \mathrm{C}$ with an initial 145 temperature ramp of $1{ }^{\circ} \mathrm{C} / \mathrm{min}$ for 5 minutes and was subsequently set to $5.63^{\circ} \mathrm{C} / \mathrm{min}$, to a final 146 temperature of $330^{\circ} \mathrm{C}$ and held for 10 minutes. The ion source was set to $70 \mathrm{eV}$ and $230^{\circ} \mathrm{C}$ while

147 the transfer line to the mass spectrometer was set to $330^{\circ} \mathrm{C}$. The detector, set to full scan, monitored 148 a mass range of $m / z 45-600$ at 1 scan per second. The carrier gas, helium, was set at a flow rate of $1491 \mathrm{ml} / \mathrm{min}$.

\section{Data Analysis}

152 Due to co-elution, the peaks of the ${ }^{13} \mathrm{C}_{6}$-sorbitol internal standard and glutamate could not be 153 deconvoluted. Results were therefore normalized using the total ion chromatogram (TIC). The presence of aberrant peaks suggested that two of the 6-well plates (1 Hz stimulation condition)

155 were not adequately washed during the extraction process and these were therefore excluded from 156 the final analysis. 
158 GC-MS data were viewed with AnalyzerPro v2.70 (SpectralWorks, Runcorn, UK). The mass

159 spectra of peaks from the chromatogram were matched against the NIST (National Institute of 160 Standards and Technology) mass spectral library. Metabolites with a similarity index of more than $16160 \%$ were tentatively identified as metabolites. Metabolites with multiple derivative peaks were 162 summed and treated as a single metabolite. For the peak area matrix, features that occurred in less 163 than $80 \%$ of all samples were removed, unless they were unique to one treatment group in $>75 \%$ 164 of replicates. Peak areas were normalised to the total ion chromatogram (TIC) and imported into 165 Unscrambler X version 10.1 (CAMO Software, Oslo, Norway). The data was log transformed $166[\mathrm{X}=\log (\mathrm{x}+1)]$ and a principal component analysis (PCA) was performed using a non-iterative 167 partial least squares algorithm, cross validation and no rotation. Statistical comparisons between 168 treatment samples were conducted with SPSS v21 (IBM, Corporation, Armonk, USA) using a one169 way ANOVA with Tukey's post-hoc test. 


\section{RESULTS}

173 Based on GC-MS profiling, a total of 18 reproducible intracellular metabolites were identified

174 from the total ion chromatograms (TIC) (Table 1). At $1 \mathrm{~Hz}$, significant decreases were observed 175 in a total of 12 metabolites. This included 7 amino acids, namely alanine, glycine, isoleucine, 176 phenylalanine, serine, threonine (all $\mathrm{p}<0.01)$ and aspartate (all $\mathrm{p}<0.05)$. Significant decreases

177 were also observed in cholesterol, glycylglutamic acid, inositol, pyroglutamate and succinate (all $178 \mathrm{p}<0.01)$. Non-significant decreases were observed in three carbohydrates: fructose, galactose and 179 glucose.

At $10 \mathrm{~Hz}$, significant decreases were observed in a total of 9 metabolites, all of which were also significantly decreased at $1 \mathrm{~Hz}$. These were the amino acids glycine, phenylalanine (both $\mathrm{p}<0.01$ ), isoleucine, serine and threonine (all $\mathrm{p}<0.05)$, as well as glycylglutamic acid $(\mathrm{p}<0.01)$, cholesterol, pyroglutamate and succinate (all $\mathrm{p}<0.05)$. Non-significant decreases were also observed in the same three carbohydrates as for $1 \mathrm{~Hz}$. Measured as a fold change, the decrease observed at $1 \mathrm{~Hz}$ was greater than at $10 \mathrm{~Hz}$ for all 12 significantly different metabolites.

In order to visualise the metabolomic data, relative peak areas (as measured by GC-MS) were subjected to principal component analysis (PCA). The PCA scores plot could be separated into 3 groups based on the frequency at which the cells were stimulated (Figure 2). The majority of the variation between control, $1 \mathrm{~Hz}$ and $10 \mathrm{~Hz}$ could be attributed to principal component 1 (PC-1), which accounted for $63 \%$ of the variance. 


\section{DISCUSSION}

196 This study demonstrates for the first time that in vitro application of LI-rTMS depletes selected

197 metabolites in B50 cells, including numerous amino acids. Furthermore, we confirm that LI-rMS

198 effects are frequency-specific with $1 \mathrm{~Hz}$ stimulation having stronger effects than $10 \mathrm{~Hz}$.

LI-rTMS induces specific metabolic changes associated with GABA synthesis

201

202

203

204

205

206

207

208

Following LI-rTMS, we did not detect any significant change in the levels of glucose, fructose or galactose, which are key energy supplies to the cell. This is in contrast with outcomes of high intensity rTMS in human and in cat studies, where changes in glucose levels were detected during and immediately after stimulation [Valero-Cabré et al., 2007]. In the latter studies, glucose uptake was significantly reduced during stimulation, suggesting a local suppression of neuronal firing [Valero-Cabré et al., 2007]. However, immediately after high frequency, high intensity rTMS, the same regions had increased metabolism [Valero-Cabré et al., 2005], presumably due to long lasting plastic changes in intracortical circuitry [Walsh and Pascual-Leone, 2003]. Together, these findings suggest that the intensity and frequency of stimulation contribute to changes in metabolic response.

However, in contrast to the stability of major carbohydrates, the metabolic profiles of metabolites implicated in GABA synthesis were reduced following LI-rMS. GABA is synthesised via glutamate, from $\alpha$-ketoglutarate, a principal component of the TCA cycle (Figure 3, box a). We observed depletion of three amino acids involved in de novo synthesis of TCA cycle intermediates, aspartate, phenylalanine and isoleucine (see Figure 3, boxes b, c and d, respectively). A possible explanation is that LI-rMS caused increased spontaneous neurotransmitter release in B50 cells, 
218 requiring the use of $\alpha$-ketoglutarate to replenish GABA pools and depleting the downstream TCA

219 cycle intermediates and their substrates (Table 1 and yellow boxes, Figure 3). Similarly, increased

220 GABA synthesis could explain the LI-rTMS dependent reduction in pyroglutamate and alanine

221 [Kumar and Bachhawat, 2012; Westergaard et al., 1993], both of which can be converted to

222 glutamate and subsequently to GABA. In support of this, spontaneous transmitter release is

223 increased following LI-rMS in excitatory neurons [Ahmed and Wieraszko, 2008], and our results

224 suggest a similar increase in spontaneous GABA release from B50 cells.

\section{Reduction in other amino acids and metabolites}

227 The amino acids serine and glycine were also significantly decreased following $1 \mathrm{~Hz}$ and $10 \mathrm{~Hz}$

228 LI-rMS. The technique used in our study could not differentiate between L- and D-serine.

229 Although both stereoisomers are found in the brain, only L-serine is incorporated into proteins,

230 while D-serine acts as a neuromodulator by co-activating NMDA receptors [Wolosker, 2006]. The

231 reduction in serine could be due to increased protein synthesis, which has been shown for specific

232 proteins such as BDNF, c-fos and various neurotransmitter receptors following rTMS

233 [Chervyakov et al., 2015; Rodger et al., 2012]. Glycine is mostly synthesized de novo in the brain

234 from serine, rather than taken up via the blood-brain barrier [Shank and Aprison, 1970], and

235 accumulation of glycine in neurons requires the activity of the glycine transporter GlyT2 [Gomeza

236 et al., 2003]. Thus, disruption of L-serine transport and/or diminished conversion of L-serine into

237 glycine may contribute to the observed reduction in glycine. A key function for serine and glycine

238 is to act as co-agonists for the NMDA receptor, which plays a central role in the long term plastic

239 changes induced by high intensity rTMS[Vlachos et al., 2012], and may also contribute to LI-

240 rTMS effects [Makowiecki et al., 2014; Rodger et al., 2012]. 
LI-rMS downregulates inositol and cholesterol - implications for calcium signalling and

244 exocytosis

245 Inositol plays a key role as a precursor for inositol lipid and inositol phosphate syntheses, which

246 are vital for signal transduction and intracellular calcium homeostasis [Wen et al., 2011]. A

247 previous study showed that LI-rMS increases the levels of intracellular calcium in cortical neurons

248 by release from intracellular stores, possibly via inositol-1,4,5-triphosphate (IP3) signalling [Grehl

249 et al., 2015]. Inositol depletion as detected here may thus reflect the changes in intracellular

250 signalling events induced by LI-rMS. Cholesterol was also decreased in our study. This lipid is not

251 only important as a structural constituent of lipid rafts and cell membranes, but also modulates

252 vesicle trafficking and exocytosis [Churchward et al., 2005; Lang et al., 2001; Zhang et al., 2009 ].

253 A recent study in ageing mice found that rTMS reduced cholesterol that accumulated with age

254 [Wang et al., 2013]. This is important because cholesterol is associated with oxidative stress during

255 normal aging [Cutler et al., 2004], and decreasing cholesterol levels can improve cognition in rats

256 [Wang et al., 2013]. A decrease of cholesterol following rTMS and LI-rTMS, as suggested by our

257 data, may thus contribute to the cognitive improvements observed following rTMS treatment of

258 Alzheimer's patients [Hsu et al., 2015], and also in healthy volunteers [Miniussi and Ruzzoli, 259 2013].

Frequency-dependent metabolic changes

262 High and low frequency rTMS have different effects on neuronal circuits, with functional and 263 genetic studies identifying distinct mechanisms in different neuronal cell types [Grehl et al., 2015; 
264 Pell et al., 2011]. Previous work using high intensity rTMS in anaesthetised cats demonstrated that

265 metabolic changes were also frequency specific with increased glucose metabolism following

266 high, but not low frequency stimulation [Valero-Cabré et al., 2007]. Our study provides a different

267 approach, because we examined metabolic changes in a uniform population of neuronal cells with

268 an inhibitory phenotype. Thus our finding that the effects of LI-rMS are stronger with $1 \mathrm{~Hz}$ than

269 with $10 \mathrm{~Hz}$ matches the inhibitory effect of $1 \mathrm{~Hz}$ that is consistently reported in animal and human

270 literature [Chen et al., 1997; Maeda et al., 2000; Pell et al., 2011; Trippe et al., 2009]. However,

271 because LI-rTMS does not induce action potentials, it remains unclear whether the metabolic

272 changes we report are a direct consequence of the electromagnetic field, or whether they are

273 secondary to the changes in excitability induced by rTMS.

274 B50 neuroblastoma cells are derived from the rat central nervous system [Schubert et al., 1974]

275 and have been used extensively to study the pathways involved in cell death, proliferation and

276 migration (eg. Honma et al., 1996). Although cells grown in culture remain a highly simplified

277 model of the CNS (discussed in Grehl et al., 2015), B50 cells have been reported to fire regenerative

278 action potentials, and contain high levels of the GABA bioythetic enzyme, glutamate decarboxylase

279 (GAD), as well as GABA itself, suggesting that they retain some key functional features of inhibitory

280 neurons [Schubert et al., 1974]. Future studies in large, uniform populations of neuronal-like cells

281 will contribute to our understanding not only of the mechanism whereby cells detect

282 electromagnetic fields, but also of the signalling events such as kinase and phosphatase cascades,

283 that underpin the complex cellular responses such as apoptosis, differentiation, migration and 284 proliferation.

285

286 CONCLUSION 
287 Our study showed that LI-rMS induces a depletion of metabolites that suggested higher 288 spontaneous activity may have resulted in an increase in GABA release. While the B50 cell line is 289 composed of cells that are electrically excitable and homogenous thus allowing for a targeted 290 examination of the effects of LI-rMS on a defined cell population, the absence of defined neural 291 circuits and other cellular contributors (e.g. excitatory neurons and glia) limits the degree to which 292 our results can be extrapolated to the human brain. Nonetheless, the changes we describe provide 293 novel insights into how LI-rTMS may modulate neural tissue and contribute to our understanding 294 of the therapeutic application of electromagnetic brain stimulation.

295

296

297

298

299

300

301

302

\section{ACKNOWLEDGEMENTS}

The authors are grateful to Dr Alex Tang for advice and assistance with magnetic field measurements, to Dr Kartik Iyer for feedback on the manuscript, and to Mrs Marissa PenroseMenz for assisting with figure preparation. 


\section{REFERENCES}

Abbiss H, Maker GL, Gummer J, Sharman MJ, Phillips JK, Boyce M, Trengove RD. 2012. Development of a non-targeted metabolomics method to investigate urine in a rat model of polycystic kidney disease. Nephrology 17:104-110.

Ahmed Z, Wieraszko A. 2008. The mechanism of magnetic field-induced increase of excitability in hippocampal neurons. Brain Research 1221:30-40.

Aldinucci C, Palmi M, Sgaragli G, Benocci A, Meini A, Pessina F, Pessina GP. 2000. The effect of pulsed electromagnetic fields on the physiologic behaviour of a human astrocytoma cell line. Biochimica et biophysica acta 1499:101-108.

Balassa T, Varro P, Elek S, Drozdovszky O, Szemerszky R, Vilagi I, Bárdos G. 2013. Changes in synaptic efficacy in rat brain slices following extremely low-frequency magnetic field exposure at embryonic and early postnatal age. International Journal of Developmental Neuroscience 31:724-730.

Barker AT, Jalinous R, Freeston IL. 1985. Non-invasive magnetic stimulation of human motor cortex. Lancet 1:1106-1107.

Chen R, Classen J, Gerloff C, Celnik P, Wassermann EM, Hallett M, Cohen LG. 1997. Depression of motor cortex excitability by low-frequency transcranial magnetic stimulation. Neurology 48:1398-1403.

Capone F, Dileone M, Profice P, Pilato F, Musumeci G, Minicuci G, Ranieri F, Cadossi R, Setti S, Tonali PA, Di Lazzaro V. 2009. Does exposure to extremely low frequency magnetic fields produce functional changes in human brain? Journal of Neural Transmission 116:257-265.

Chervyakov A, Sinitsyn D, Chernyavsky A, Piradov M. 2015. Possible mechanisms underlying the therapeutic effects of transcranial magnetic stimulation. Frontiers in Human Neuroscience 9.

Churchward MA, Rogasevskaia T, Höfgen J, Bau J, Coorssen JR. 2005. Cholesterol facilitates the native mechanism of Ca2+-triggered membrane fusion. Journal of Cell Science 118:4833-4848.

Cutler RG, Kelly J, Storie K, Pedersen WA, Tammara A, Hatanpaa K, Troncoso JC, Mattson MP. 2004. Involvement of oxidative stress-induced abnormalities in ceramide and cholesterol metabolism in brain aging and Alzheimer's disease. Proceedings of the National Academy of Sciences of the United States of America 101:2070-2075.

Davey KR, Riehl M. 2006. Suppressing the surface field during transcranial magnetic stimulation. Biomedical Engineering, IEEE Transactions on 53:190-194.

Di Lazzaro V, Capone F, Apollonio F, Borea PA, Cadossi R, Fassina L, Grassi C, Liberti M, Paffi A, Parazzini $M$ and others. 2013. A Consensus Panel Review of Central Nervous System Effects of the Exposure to Low-Intensity Extremely Low-Frequency Magnetic Fields. Brain Stimulation 6:469476.

George MS, Lisanby SH, Avery D, McDonald WM, Durkalski V, Pavlicova M, Anderson B, Nahas Z, Bulow P, Zarkowski P, Holtzheimer III PE, Schwartz T, Sackeim HA. 2010. Daily left prefrontal transcranial magnetic stimulation therapy for major depressive disorder: A sham-controlled randomized trial. Archives of General Psychiatry 67:507-516.

Gomeza J, Ohno K, Hülsmann S, Armsen W, Eulenburg V, Richter DW, Laube B, Betz H. 2003. Deletion of the Mouse Glycine Transporter 2 Results in a Hyperekplexia Phenotype and Postnatal Lethality. Neuron 40:797-806.

GrehI S, Viola HM, Fuller-Carter PI, Carter KW, Dunlop SA, Hool LC, Sherrard RM, Rodger J. 2015. Cellular and Molecular Changes to Cortical Neurons Following Low Intensity Repetitive Magnetic Stimulation at Different Frequencies. Brain Stimulation 8:114-123.

Hoffman RE, Boutros NN, Hu S, Berman RM, Krystal JH, Charney DS. 2000. Transcranial magnetic stimulation and auditory hallucinations in schizophrenia. Lancet 355:1073-1075. 
349

350

351

352

353

354

355

356

357

358

359

360

361

362

363

364

365

366

367

368

369

370

371

372

373

374

375

376

377

378

379

380

381

382

383

384

385

386

387

388

389

390

391

392

393

394

395

396

Honma N1, Uchida A, Hirose H, Srsen V, Kishimoto T, Hisanaga S. 1996. Two types of apoptotic cell death of rat central nervous system-derived neuroblastoma B50 and B104 cells: apoptosis induced during proliferation and after differentiation. J Neurochem. 67:1856-65.

Hsu W-Y, Ku Y, Zanto TP, Gazzaley A. 2015. Effects of noninvasive brain stimulation on cognitive function in healthy aging and Alzheimer's disease: a systematic review and meta-analysis. Neurobiology of Aging 36:2348-2359.

International Commission on Non-lonizing Radiation Protection (ICNIRP). 1998. Guidelines for limiting exposure to time-varying electric, magnetic, and electromagnetic fields (up to $300 \mathrm{GHz}$ ). Health Phys. 74:494-522.

Kumar A, Bachhawat AK. 2012. Pyroglutamic acid: throwing light on a lightly studied metabolite. Current Science (00113891) 102:288-297.

Lang T, Bruns D, Wenzel D, Riedel D, Holroyd P, Thiele C, Jahn R. 2001. SNAREs are concentrated in cholesterol - dependent clusters that define docking and fusion sites for exocytosis.

Li C-T, Chen M-H, Juan C-H, Huang H-H, Chen L-F, Hsieh J-C, Tu P-C, Bai Y-M, Tsai S-J, Lee Y-C and others. 2014. Efficacy of prefrontal theta-burst stimulation in refractory depression: a randomized sham-controlled study. Brain 137:2088-2098.

Lipton RB, Dodick DW, Silberstein SD, Saper JR, Aurora SK, Pearlman SH, Fischell RE, Ruppel PL, Goadsby PJ. 2010. Single-pulse transcranial magnetic stimulation for acute treatment of migraine with aura: a randomised, double-blind, parallel-group, sham-controlled trial. The Lancet. Neurology 9:373-380.

Maeda F, Keenan JP, Tormos JM, Topka H, Pascual-Leone A. 2000. Modulation of corticospinal excitability by repetitive transcranial magnetic stimulation. Clinical neurophysiology : official journal of the International Federation of Clinical Neurophysiology 111:800-805.

Makowiecki K, Harvey AR, Sherrard RM, Rodger J. 2014. Low-Intensity Repetitive Transcranial Magnetic Stimulation Improves Abnormal Visual Cortical Circuit Topography and Upregulates BDNF in Mice. The Journal of Neuroscience 34:10780-10792.

Manikonda PK, Rajendra P, Devendranath D, Gunasekaran B, Channakeshava, Aradhya RS, Sashidhar RB, Subramanyam C. 2007. Influence of extremely low frequency magnetic fields on Ca2+ signaling and NMDA receptor functions in rat hippocampus. Neuroscience Letters 413:145-149.

Mantovani A, Simpson HB, Fallon BA, Rossi S, Lisanby SH. 2010. Randomized sham-controlled trial of repetitive transcranial magnetic stimulation in treatment-resistant obsessive-compulsive disorder. The International Journal of Neuropsychopharmacology 13:217-227.

Martiny K, Lunde M, Bech P. 2010. Transcranial low voltage pulsed electromagnetic fields in patients with treatment-resistant depression. Biological psychiatry 68:163-169.

Miniussi C, Ruzzoli M. 2013. Chapter 56 - Transcranial stimulation and cognition. In: Andres ML, Mark H (eds.). Handbook of Clinical Neurology: Elsevier. pp 739-750.

Müller-Dahlhaus F, Vlachos A. 2013. Unraveling the cellular and molecular mechanisms of repetitive magnetic stimulation. Frontiers in Molecular Neuroscience 6:50.

Navarro EA, Gomez-Perretta C, Montes F. 2016. Low intensity magnetic field influences short-term memory: A study in a group of healthy students. Bioelectromagnetics 37:37-48.

O'Reardon JP, Solvason HB, Janicak PG, Sampson S, Isenberg KE, Nahas Z, McDonald WM, Avery D, Fitzgerald PB, Loo C and others. 2007. Efficacy and Safety of Transcranial Magnetic Stimulation in the Acute Treatment of Major Depression: A Multisite Randomized Controlled Trial. Biological Psychiatry 62:1208-1216.

Pell GS, Roth Y, Zangen A. 2011. Modulation of cortical excitability induced by repetitive transcranial magnetic stimulation: influence of timing and geometrical parameters and underlying mechanisms. Progress in neurobiology 93:59-98.

PeerJ reviewing PDF | (2018:01:23205:1:1:NEW 17 Feb 2018) 
Plewnia C, Reimold M, Najib A, Reischl G, Plontke SK, Gerloff C. 2007. Moderate therapeutic efficacy of positron emission tomography-navigated repetitive transcranial magnetic stimulation for chronic tinnitus: a randomised, controlled pilot study. Journal of Neurology, Neurosurgery \& Psychiatry 78:152-156.

Rodger J, Mo C, Wilks T, Dunlop SA, Sherrard RM. 2012. Transcranial pulsed magnetic field stimulation facilitates reorganization of abnormal neural circuits and corrects behavioral deficits without disrupting normal connectivity. FASEB journal : official publication of the Federation of American Societies for Experimental Biology 26:1593-1606.

Rohan ML, Yamamoto RT, Ravichandran CT, Cayetano KR, Morales OG, Olson DP, Vitaliano G, Paul SM, Cohen BM. 2014. Rapid Mood-Elevating Effects of Low Field Magnetic Stimulation in Depression. Biological Psychiatry 76:186-193.

Rudiak D, Marg E. 1994. Finding the depth of magnetic brain stimulation: a re-evaluation. Electroencephalography and Clinical Neurophysiology/Evoked Potentials Section 93:358-371.

Shahbazi-Gahrouei D, Shiri L, Alaei H, Naghdi N. 2016. The effect of continuous ELF-MFs on the level of 5-HIAA in the raphe nucleus of the rat. Journal of Radiation Research doi:10.1093/jrr/rrv093.

Shank RP, Aprison MH. 1970. The metabolism in vivo of glycine and serine in eight areas of the rat central nervous system. Journal of Neurochemistry 17:1461-1475.

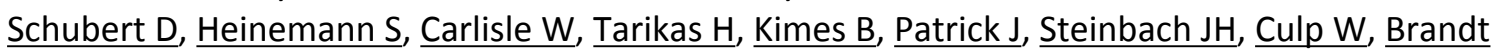
BL. 1974. Clonal cell lines from the rat central nervous system. Nature 249:224-7. Shupak NM, Prato FS, Thomas AW. 2004. Human exposure to a specific pulsed magnetic field: effects on thermal sensory and pain thresholds. Neuroscience letters 363:157-162.

Terao Y, Ugawa Y. 2002. Basic Mechanisms of TMS. Journal of Clinical Neurophysiology 19:322-343.

Trippe J, Mix A, Aydin-Abidin S, Funke K, Benali A. 2009. $\theta$ burst and conventional low-frequency rTMS differentially affect GABAergic neurotransmission in the rat cortex. Experimental brain research. Experimentelle Hirnforschung. Expérimentation cérébrale 199:411-421.

Valero-Cabré A, Payne B, Pascual-Leone A. 2007. Opposite impact on 14C-2-deoxyglucose brain metabolism following patterns of high and low frequency repetitive transcranial magnetic stimulation in the posterior parietal cortex. Experimental Brain Research 176:603-615.

Valero-Cabré A, Payne BR, Rushmore J, Lomber SG, Pascual-Leone A. 2005. Impact of repetitive transcranial magnetic stimulation of the parietal cortex on metabolic brain activity: a 14C-2DG tracing study in the cat. Experimental brain research. Experimentelle Hirnforschung. Expérimentation cérébrale 163:1-12.

Vlachos A, Müller-Dahlhaus F, Rosskopp J, Lenz M, Ziemann U, Deller T. 2012. Repetitive magnetic stimulation induces functional and structural plasticity of excitatory postsynapses in mouse organotypic hippocampal slice cultures. The Journal of neuroscience : the official journal of the Society for Neuroscience 32:17514-17523.

Walleczek J, Budinger TF. 1992. Pulsed magnetic field effects on calcium signaling in lymphocytes: dependence on cell status and field intensity. FEBS letters 314:351-355.

Walsh V, Pascual-Leone A. 2003. Transcranial magnetic stimulation: a neurochronometrics of mind: MIT press Cambridge, MA.

Wang H, Geng Y, Han B, Qiang J, Li X, Sun M, Wang Q, Wang M. 2013. Repetitive Transcranial Magnetic Stimulation Applications Normalized Prefrontal Dysfunctions and Cognitive-Related Metabolic Profiling in Aged Mice. PLoS ONE 8:e81482.

Wen PJ, Osborne SL, Meunier FA. 2011. Dynamic control of neuroexocytosis by phosphoinositides in health and disease. Progress in Lipid Research 50:52-61.

Westergaard N, Varming T, Peng L, Sonnewald U, Hertz L, Schousboe A. 1993. Uptake, release and metabolism of alaume in neurons and astrocytes in primary cultures. Journal of Neuroscience Research 35:540-545. 
445 Wolosker H. 2006. D-Serine Regulation of NMDA Receptor Activity. Science Signaling 2006:pe41-pe41. 446 Yang G, Ren Z, Mei YA. 2015. Exposure to $50 \mathrm{~Hz}$ magnetic field modulates GABAA currents in cerebellar granule neurons through an EP receptor-mediated PKC pathway. Journal of Cellular and Molecular Medicine 19:2413-2422.

Zhang J, Xue R, Ong W-Y, Chen P. 2009. Roles of Cholesterol in Vesicle Fusion and Motion. Biophysical Journal 97:1371-1380.

Zhang X, Liu X, Pan L, Lee I. 2010. Magnetic fields at extremely low-frequency $(50 \mathrm{~Hz}, 0.8 \mathrm{mT})$ can induce the uptake of intracellular calcium levels in osteoblasts. Biochemical and Biophysical Research Communications 396:662-666. 
FIGURE LEGENDS

456 Figure 1.Stimulation equipment. A,B: Photographs of the in vitro stimulation coils used in this

457 study. Views are from the side (A) and top (B). The coils were situated at a distance of $2.8 \mathrm{~mm}$

458 from the bottom of the culture well because of the thickness of the coverplate and base of the

459 culture dish.C: heatmap showing the measured change in magnetic field for a single pulse (dB/dT)

460 when 6 coils are placed in a 6-well plate arrangement. Note the lack of overlap of magnetic field

461 between the coils/wells of the plates.

462

463

Figure 2. Principal component analysis score plot of intracellular metabolite profiles (each data

point represents a replicate sample) from GC-MS analysis of cells stimulated with LI-rMS at $1 \mathrm{~Hz}$ $(\mathrm{n}=4), 10 \mathrm{~Hz}(\mathrm{n}=6)$ and unstimulated controls $(\mathrm{n}=6)$.

466

467

468

Figure 3. TCA cycle. Modified TCA cycle showing effects of LI-rMS on metabolic profile of B50 cells. Metabolites highlighted in yellow were significantly reduced in B50 cells following 1 and/or $10 \mathrm{~Hz}$ LI-rMS, compared to control B50 cells. The level of glucose (highlighted in green) was measured in our experiments and found to be unchanged by LI-rMS. The observed changes are proposed to result from increased de novo GABA synthesis (pathway a) that results in depletion of TCA cycle intermediates and precursors (pathways b, c, d). Suspected point of interference by LI-rMS is noted with ' $\mathrm{X}$ '.

Table 1. Intracellular metabolites identified by GC-MS and PCA as contributing the most to the

476 variance between unstimulated controls and cells stimulated at 1 or $10 \mathrm{~Hz}$ and the fold change

477 observed between stimulated cells and controls $1 \mathrm{~Hz}(\mathrm{n}=4), 10 \mathrm{~Hz}(\mathrm{n}=6)$ and unstimulated controls 
478 ( $n=6)$. Statistical significance was determined using a one-way ANOVA with Tukey's post-hoc

479 test and is indicated as $*=\mathrm{p} \leq 0.05 ; * *=\mathrm{p} \leq 0.01$. 


\section{Figure 1}

Photographs showing equipment used for magnetic stimulation of cells

Stimulation equipment. $A, B$ : Photographs of the in vitro stimulation coils used in this study.

Views are from the side (A) and top (B). The coils were situated at a distance of $2.8 \mathrm{~mm}$ from the bottom of the culture well because of the thickness of the coverplate and base of the culture dish.C: heatmap showing the measured change in magnetic field for a single pulse $(\mathrm{dB} / \mathrm{dT})$ when 6 coils are placed in a 6 -well plate arrangement. Note the lack of overlap of magnetic field between the coils/wells of the plates. 

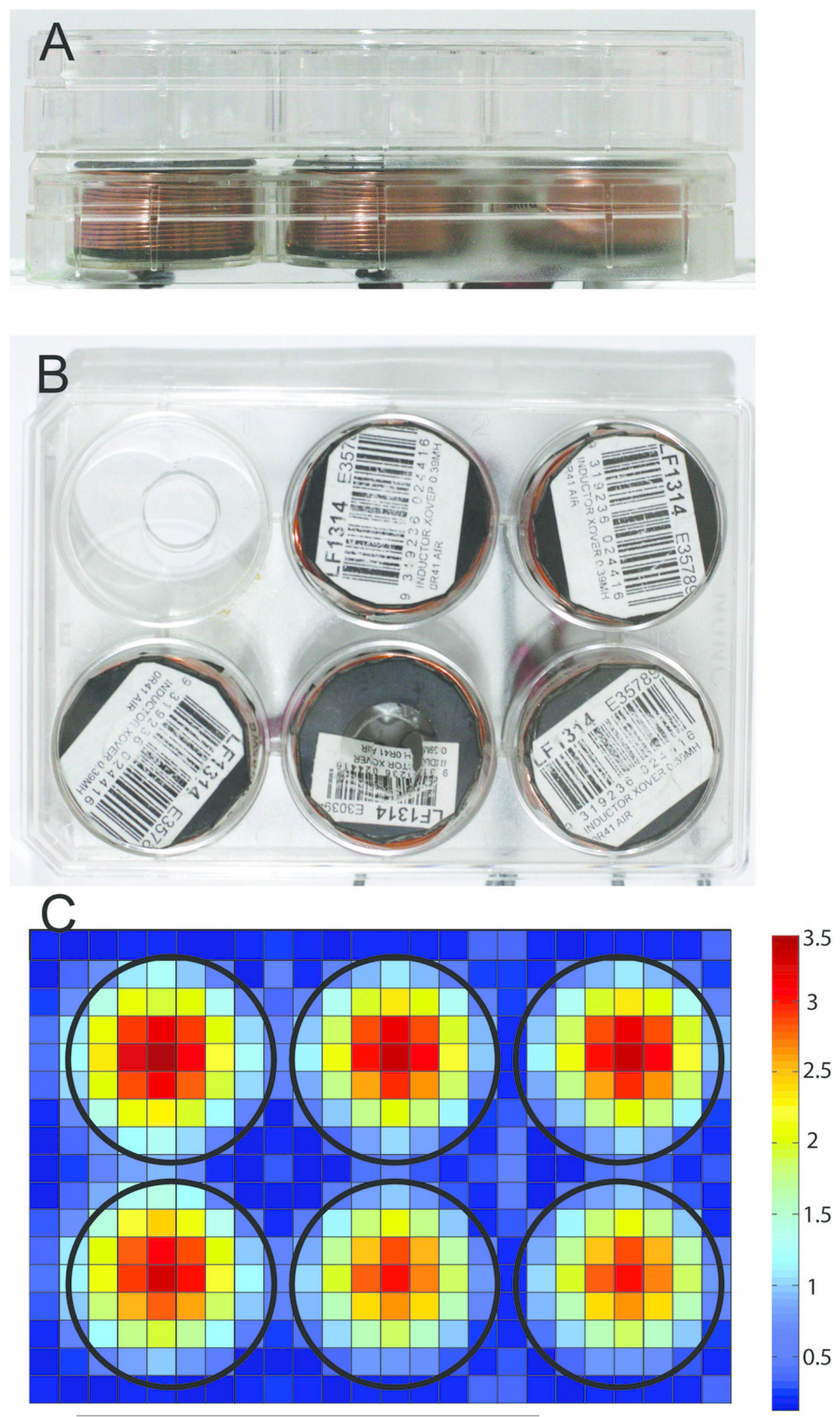
Figure 2

Principal component analysis of metabolic profiles

Principal component analysis score plot of intracellular metabolite profiles (each data point represents a replicate sample) from GC-MS analysis of cells stimulated with LI-rMS at $1 \mathrm{~Hz}$ $(n=4), 10 \mathrm{~Hz}(n=6)$ and unstimulated controls $(n=6)$.

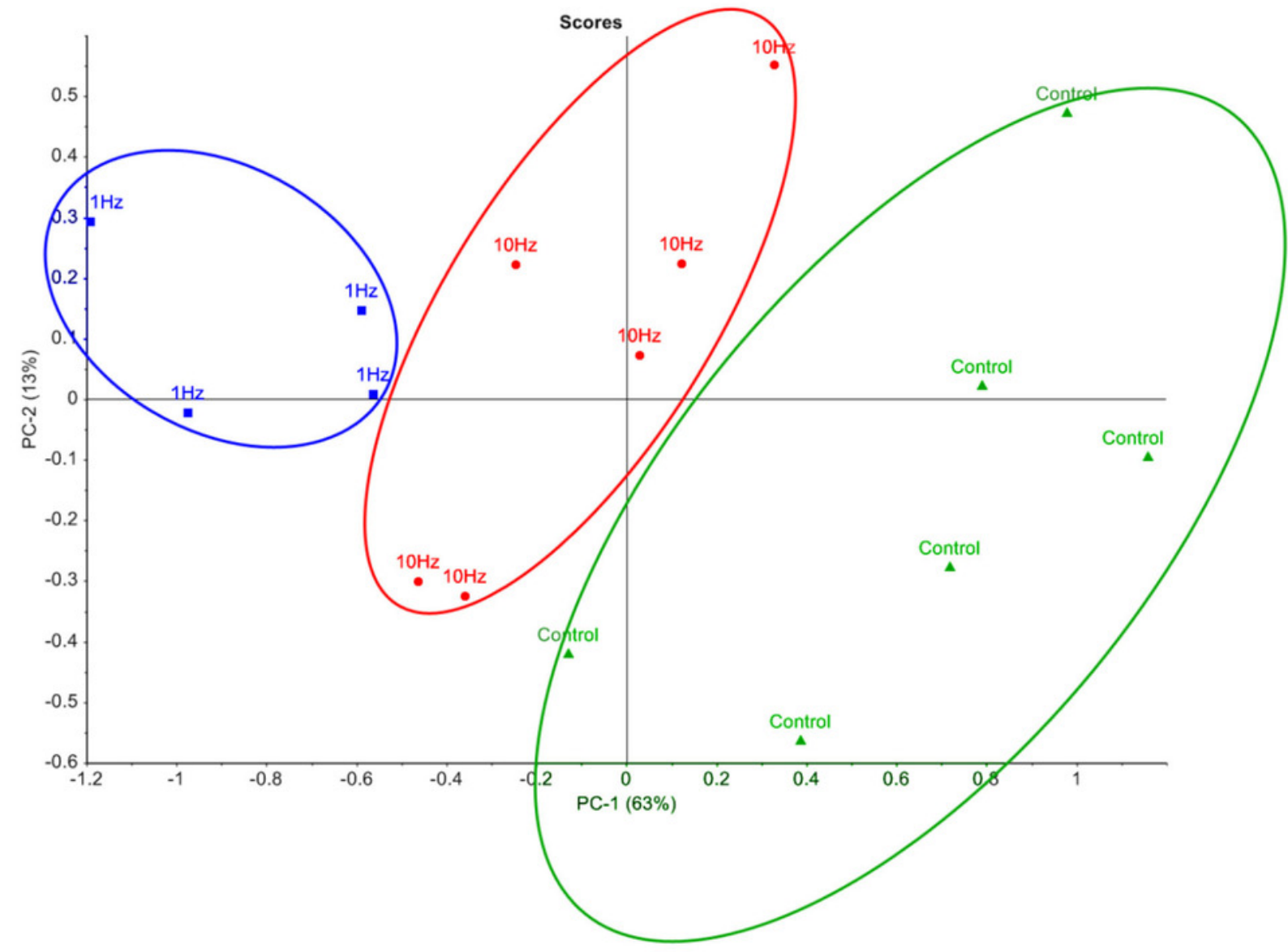




\section{Figure 3}

Modified TCA cycle showing effects of LI-rMS on metabolic profile of B50 cells

Metabolites highlighted in yellow were significantly reduced in B50 cells following 1 and/or 10 $\mathrm{Hz}$ LI-rMS, compared to control B50 cells. The level of glucose (highlighted in green) was measured in our experiments and found to be unchanged by LI-rMS. The observed changes are proposed to result from increased de novo GABA synthesis (pathway a) that results in depletion of TCA cycle intermediates and precursors (pathways b, c, d). Suspected point of interference by LI-rMS is noted with ' $X$ '. 


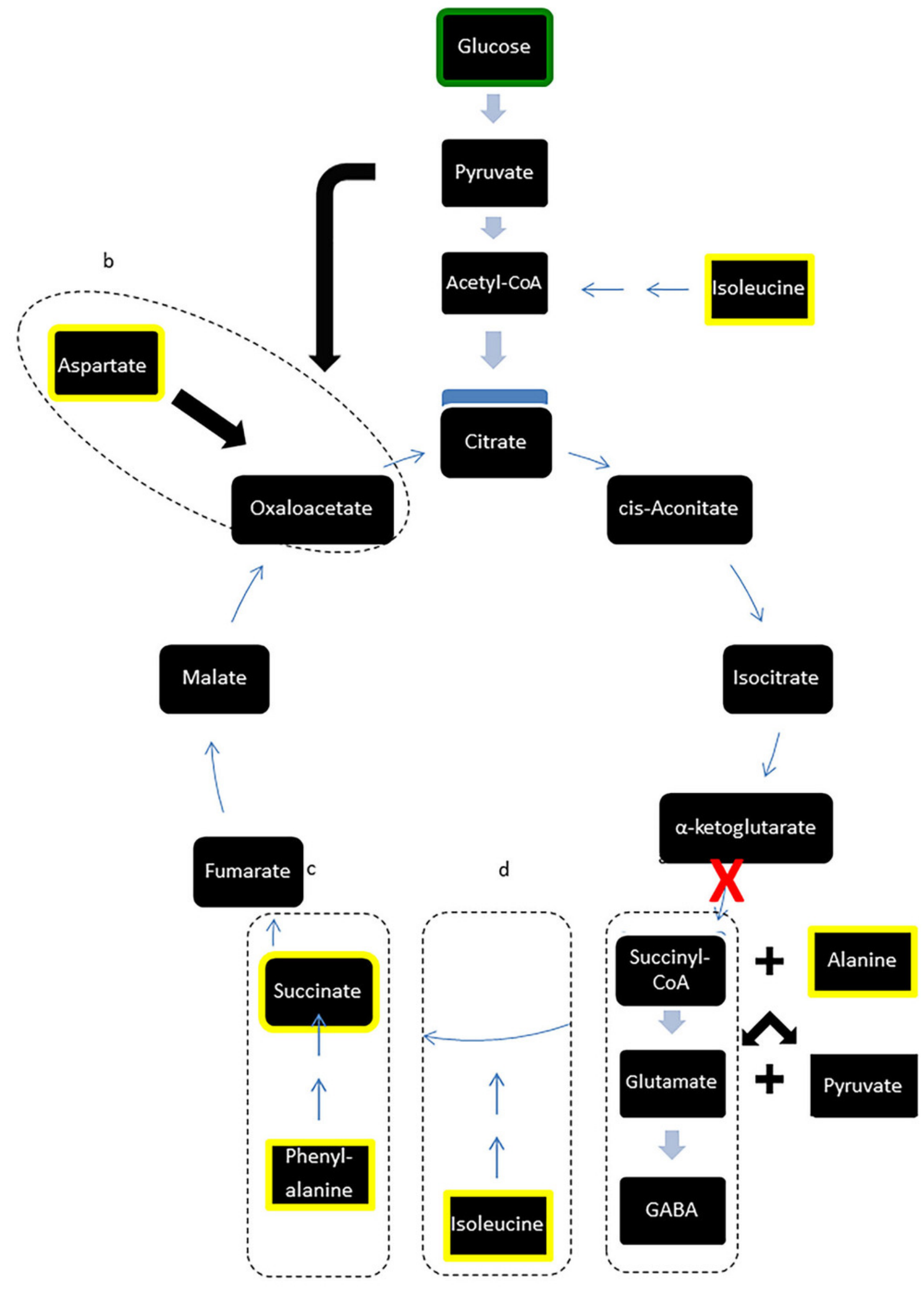




\section{Table $\mathbf{1}$ (on next page)}

Fold change of metabolites following magnetic stimulation

Intracellular metabolites identified by GC-MS and PCA as contributing the most to the variance between unstimulated controls and cells stimulated at 1 or $10 \mathrm{~Hz}$ and the fold change observed between stimulated cells and controls $1 \mathrm{~Hz}(n=4), 10 \mathrm{~Hz}(n=6)$ and unstimulated controls $(n=6)$. Statistical significance was determined using a one-way ANOVA with Tukey's post-hoc test and is indicated as $*=p \leq 0.05 ; * *=p \leq 0.01$. 
Manuscript to be reviewed

\begin{tabular}{|c|c|c|c|}
\hline Metabolite & Fold change $(1 \mathrm{~Hz})$ & Fold change (10 Hz) & F-statistic \\
\hline \multicolumn{4}{|l|}{ Amino acids } \\
\hline Alanine & $0.625^{* *}$ & 0.873 & 6.477 \\
\hline Aspartate & $0.322^{*}$ & 0.619 & 3.846 \\
\hline Glycine & $0.570^{* *}$ & $0.761 * *$ & 19.162 \\
\hline Isoleucine & $0.599^{* *}$ & $0.753^{*}$ & 7.835 \\
\hline Phenylalanine & $0.379 * *$ & $0.477 * *$ & 10.710 \\
\hline Serine & $0.389^{* *}$ & $0.656^{*}$ & 15.784 \\
\hline Threonine & $0.606^{* *}$ & $0.764 *$ & 11.286 \\
\hline Valine & 0.942 & 0.956 & 0.064 \\
\hline \multicolumn{4}{|l|}{ Carbohydrates } \\
\hline Fructose & 0.638 & 0.958 & 1.788 \\
\hline Galactose & 0.629 & 0.948 & 1.149 \\
\hline Glucose & 0.858 & 0.938 & 0.161 \\
\hline \multicolumn{4}{|l|}{ Other metabolites } \\
\hline Carbonic acid, 4- & & & \\
\hline $\begin{array}{l}\text { methylphenyl phenyl } \\
\text { ester }\end{array}$ & 1.268 & 1.267 & 2.021 \\
\hline Cholesterol & $0.713^{* *}$ & $0.831^{*}$ & 8.909 \\
\hline Glycerol-3-phosphate & 0.801 & 0.798 & 4.155 \\
\hline Glycylglutamic acid & $0.526^{* *}$ & $0.693 * *$ & 26.740 \\
\hline Inositol & $0.563^{* *}$ & 0.742 & 7.881 \\
\hline Pyroglutamate & $0.531^{* *}$ & $0.727 *$ & 9.603 \\
\hline Succinate & $0.640^{* *}$ & $0.803^{*}$ & 11.461 \\
\hline
\end{tabular}

Table 1: Fold change between intracellular metabolites of cells stimulated at 1 and $10 \mathrm{~Hz}$ vs control. *

$\mathrm{p} \leq 0.05, * * \mathrm{p} \leq 0.01$ 